\title{
METRIC DIMENSION OF COMPLETE METRIC SPACES ${ }^{1}$
}

GLENN A. BOOKHOUT

1. Introduction and results. For integers $n \geqq 3$, let $\left(X_{n}, \rho\right)$ be a metric space such that

(i) $X_{n} \subset\left(K_{n}, \rho\right)$, a compact $n$-dimensional metric space;

(ii) $X_{n}=K_{n}-\bigcup_{i=1}^{\infty} A_{i}$, where the $A_{i}$ 's are mutually disjoint and closed in $K_{n}$; and

(iii) $\mu \operatorname{dim}\left(X_{n}, \rho\right)=[n / 2]$ and $\operatorname{dim} X_{n}=n-1$.

(Here $\mu \operatorname{dim}$ denotes metric dimension, which is defined in the next section, and dim denotes covering dimension.) K. Sitnikov [8, p. 23] and K. Nagami and J. H. Roberts [6, p. 426] have constructed such spaces.

The result of the present paper is stated in the following theorem.

Theorem. For integers $n \geqq 3$, let $\left(X_{n}, \rho\right)$ be a metric space with properties (i)-(iii) above. Then there exists a complete metric $\sigma$ on $X_{n}$ equivalent to $\rho$ such that

$$
\mu \operatorname{dim}\left(X_{n}, \sigma\right) \leqq[n / 2]+1 .
$$

K. Nagami and J. H. Roberts posed the following question. Is $\mu \operatorname{dim}(X, d)=\operatorname{dim} X$ for all complete metric spaces $(X, d)$ ? In [1, p.166] Richard E. Hodel posed an analogous question. Is $d_{2}(X, d)$ $=\operatorname{dim} X$ for all complete metric spaces $(X, d)$ ? (The metric-dependent dimension function $d_{2}$ is defined in the next section.) It is known (see [6, Theorem 4, p. 422]) that $d_{2}(X, d) \leqq \mu \operatorname{dim}(X, d)$ for all metric spaces $(X, d)$. The present theorem gives a negative answer to these questions, since for $n \geqq 5$,

$$
\mu \operatorname{dim}\left(X_{n}, \sigma\right) \leqq[n / 2]+1<n-1=\operatorname{dim} X_{n} .
$$

M. Katětov $[4$, p. 166] proved that $\operatorname{dim} X \leqq 2 \mu \operatorname{dim}(X, d)$ for all nonempty metric spaces $(X, d)$. In view of this result of Katětov and the present theorem, the following problem is suggested.

PROBLEM. For integers $n \geqq 3$, do there exist complete metric spaces $\left(X_{n}, d\right)$ with $\mu \operatorname{dim}\left(X_{n}, d\right)=[n / 2]$ and $\operatorname{dim} X_{n}=n-1$ ?

Received by the editors June 16, 1969.

1 This research is part of a doctoral dissertation prepared at Duke University under the supervision of Professor J. H. Roberts and was supported in part by the National Science Foundation under grants GP-5222 and GP-5919. 
2. Definitions. In this paper three metric-dependent dimension functions are considered:

(i) metric dimension, denoted by $\mu \operatorname{dim}$;

(ii) $d_{2}$, introduced by K. Nagami and J. H. Roberts in [5, p. 602]; and

(iii) $d_{5}$, introduced by Richard E. Hodel in [3, p. 83]. Metric dimension, $d_{2}$, and $d_{5}$ are functions from the class of all metric spaces $(X, d)$ into $\{-1,0,1, \cdots ; \infty\}$. Condensed definitions of these functions restricted to nonempty metric spaces are as follows.

Definition. $\mu \operatorname{dim}(X, d)$ is the smallest integer $n$ such that for all $\epsilon>0$ there exists an open cover $\mathfrak{u}(\epsilon)$ of $X$ with (1) order $\mathcal{u}(\epsilon)$ $\leqq n+1$ and $(2)$ mesh $u(\epsilon)<\epsilon$.

Definition. $d_{2}(X, d)$ is the smallest integer $n$ such that given any $n+1$ pairs $\left\{C_{i}, C_{i}^{\prime}\right\}_{i=1}^{n+1}$ of closed sets with $d\left(C_{i}, C_{i}^{\prime}\right)>0$ for each $i$, there exist closed sets $\left\{B_{i}\right\}_{i=1}^{n+1}$ such that

(i) $B_{i}$ separates $C_{i}$ and $C_{i}^{\prime}$ in $X$ for each $i$ and

(ii) $\cap_{i=1}^{n+1} B_{i}=\varnothing$.

DEFINITION. $d_{5}(X, d)$ is the smallest integer $n$ such that given any countable number of pairs $\left\{C_{i}, C_{i}^{\prime}\right\}_{i=1}^{\infty}$ of closed sets with $d\left(C_{i}, C_{i}^{\prime}\right) \geqq \delta$ for each $i$ for some $\delta>0$, there exist closed sets $\left\{B_{i}\right\}_{i=1}^{\infty}$ such that

(i) $B_{i}$ separates $C_{i}$ and $C_{i}^{\prime}$ in $X$ for each $i$ and

(ii) order $\left\{B_{i}\right\}_{i=1}^{\infty} \leqq n$.

\section{Proof of the theorem.}

3.1. Reducing the PRoblem. Fix an integer $n \geqq 3$. Let $\left(X_{n}, \rho\right)$ be a metric space with properties (i)-(iii) above. We may assume that every $A_{i}$ is nonempty. Define

$$
\begin{gathered}
f_{i}(x)=\frac{1}{\rho\left(x, A_{i}\right)}, \quad\left(x \in K_{n}-A_{i}, i \geqq 1\right) ; \\
\alpha_{i}(x, y)=2^{-i} \cdot \frac{\left|f_{i}(x)-f_{i}(y)\right|}{1+\left|f_{i}(x)-f_{i}(y)\right|}, \quad\left(x, y \in K_{n}-A_{i}, i \geqq 1\right) ; \\
\sigma(x, y)=\rho(x, y)+\sum_{i=1}^{\infty} \alpha_{i}(x, y), \quad\left(x, y \in X_{n}\right) .
\end{gathered}
$$

It is known (see [2, Theorem 2-76, p. 85]) that $\sigma$ is a complete metric on $X_{n}$ equivalent to $\rho$.

We shall prove that $\mu \operatorname{dim}\left(X_{n}, \sigma\right) \leqq[n / 2]+1$. It is proved in [3, p. 85] that $d_{5}(X, d)=\mu \operatorname{dim}(X, d)$ for all separable metric spaces $(X, d)$. Now $X_{n}$ is separable, so it suffices to prove that $d_{5}\left(X_{n}, \sigma\right)$ $\leqq[n / 2]+1$. Let $\left\{C_{i}, C_{i}^{\prime}\right\}_{i=1}^{\infty}$ be a countable number of pairs of closed 
sets in $X_{n}$ with $\sigma\left(C_{i}, C_{i}^{\prime}\right) \geqq \epsilon$ for each $i$ for some $\epsilon>0$. We want to show that there exist closed sets $\left\{B_{i}\right\}_{i=1}^{\infty}$ in $X_{n}$ such that

(i) $B_{i}$ separates $C_{i}$ and $C_{i}^{\prime}$ in $X_{n}$ for each $i$ and

(ii) order $\left\{B_{i}\right\}_{i=1}^{\infty} \leqq[n / 2]+1$.

Since $\sum_{i=1}^{\infty} \alpha_{i}$ converges uniformly in $X_{n}$, there exists an integer $N>1$ such that $\sum_{i=N+1}^{\infty} \alpha_{i}(x, y)<\epsilon / 2$ for all $x, y \in X_{n}$. Define

$$
\begin{gathered}
\sigma^{N}(x, y)=\rho(x, y)+\sum_{i=1}^{N} \alpha_{i}(x, y), \quad\left(x, y \in X_{n}\right) . \\
A=\bigcup_{i=1}^{N} A_{i} .
\end{gathered}
$$

Then clearly $\sigma^{N}$ is a metric on $X_{n}$ equivalent to $\rho$. Also, since $\sigma\left(C_{i}, C_{i}^{\prime}\right) \geqq \epsilon$ for all $i$, it follows that

$$
\sigma^{N}\left(C_{i}, C_{i}^{\prime}\right) \geqq \frac{\epsilon}{2} \quad \text { for all } i .
$$

3.2. Definitions. Define

$$
\begin{gathered}
\delta=\min \left\{\rho\left(A_{i}, A_{j}\right): i, j \in\{1,2, \cdots, N\}, i \neq j\right\}, \\
\gamma=\min \left\{\frac{\delta}{4}, \frac{\epsilon}{6}, \frac{\epsilon \delta^{2}}{24(N-1)}\right\} .
\end{gathered}
$$

3.3. Assertion 1. For all numbers $a$ such that $0<a \leqq \delta / 4$, there exists an $\epsilon(a)>0$ such that $\rho\left(C_{i}, C_{i}^{\prime}\right) \geqq \gamma$ in $S(\epsilon(a)) \quad\left(\equiv\left\{x \in K_{n}: a-\epsilon(a)\right.\right.$ $<\rho(x, A)<a+\epsilon(a)\})$ for $i \geqq 1$.

Proof. Fix $a$ such that $0<a \leqq \delta / 4$. Choose $\epsilon(a)>0$ such that $\epsilon(a)<\min \left\{a / 2, \epsilon a^{2} / 48\right\}$. Suppose there exists an integer $i \geqq 1$ such that $\rho\left(C_{i}, C_{i}^{\prime}\right)<\gamma$ in $S(\epsilon(a))$. Then there exist points $x \in C_{i}$ and $y \in C_{i}^{\prime}$ such that $\{x, y\} \subset S(\epsilon(a))$ and $\rho(x, y)<\gamma$. From the definition of $\gamma$ and the choice of $\epsilon(a)$, it follows that $\rho(x, y)<\delta / 4, \rho(x, A)<3 \delta / 8$, and $\rho(y, A)<3 \delta / 8$. Therefore by the definition of $\delta$, there exists an integer $k \in\{1,2, \cdots, N\}$ such that $\rho\left(x, A_{k}\right)<3 \delta / 8$ and $\rho\left(y, A_{k}\right)$ $<3 \delta / 8$. Thus for $i \in\{1,2, \cdots, N\}$ and $i \neq k, \rho\left(x, A_{i}\right)>\delta / 2$ and $\rho\left(y, A_{i}\right)>\delta / 2$. It follows that $a-\epsilon(a)<\rho\left(x, A_{k}\right)<a+\epsilon(a)$ and $a-\epsilon(a)$ $<\rho\left(y, A_{k}\right)<a+\epsilon(a)$. Hence $\left|\rho\left(x, A_{k}\right)-\rho\left(y, A_{k}\right)\right|<2 \epsilon(a)$. Finally, $\rho\left(x, A_{k}\right)>a / 2$ and $\rho\left(y, A_{k}\right)>a / 2$. From the definitions of $\sigma^{N}$ and $\gamma$ and the inequalities above, it follows that 


$$
\begin{aligned}
\sigma^{N}(x, y) & \leqq \rho(x, y)+\sum_{i=1}^{N}\left|f_{i}(x)-f_{i}(y)\right| \\
& \leqq \rho(x, y)+\sum_{i=1}^{N} \frac{\left|\rho\left(x, A_{i}\right)-\rho\left(y, A_{i}\right)\right|}{\rho\left(x, A_{i}\right) \cdot \rho\left(y, A_{i}\right)} \\
& \leqq \rho(x, y)+\sum_{\substack{i=1 \\
i \neq k}}^{N} \frac{\rho(x, y)}{\rho\left(x, A_{i}\right) \cdot \rho\left(y, A_{i}\right)}+\frac{\left|\rho\left(x, A_{k}\right)-\rho\left(y, A_{k}\right)\right|}{\rho\left(x, A_{k}\right) \cdot \rho\left(y, A_{k}\right)} \\
& <\gamma+\frac{(N-1) \gamma}{\delta^{2} / 4}+\frac{2 \epsilon(a)}{a^{2} / 4} \\
& <\epsilon / 6+\epsilon / 6+\epsilon / 6=\epsilon / 2,
\end{aligned}
$$

contradicting (1).

3.4. Construction of $C_{i j}, C_{i j}^{\prime}$. Now (i) $\{S(\epsilon(a)): 0<a \leqq \delta / 4\}$ is a collection of open sets in $K_{n}$ covering $\left\{x \in K_{n}: 0<\rho(x, A) \leqq \delta / 4\right\}$ and (ii) $\left\{x \in K_{n}: \delta /\left(4 \cdot 2^{j}\right) \leqq \rho(x, A) \leqq \delta /\left(4 \cdot 2^{j-1}\right)\right\}$ is compact for $j \geqq 1$. Using (i) and (ii), it is easy to prove that there exist a sequence $\left\{a_{j}\right\}_{j=1}^{\infty}$ of positive numbers $\leqq \delta / 4$ such that

(a) $\bigcup_{j=1}^{\infty} S\left(\epsilon\left(a_{j}\right)\right)$ covers $\left\{x \in K_{n}: 0<\rho(x, A) \leqq \delta / 4\right\}$ and

(b) the sequence $\left\{a_{j}\right\}_{j=1}^{\infty}$ converges to 0 .

We can choose a sequence $\left\{\delta_{j}\right\}_{j=1}^{\infty}$ of distinct positive numbers such that $\delta_{1}=\delta / 4,\left\{\delta_{j}\right\}_{j=1}^{\infty}$ is a strictly decreasing sequence converging to 0 , and for each $j \geqq 2$ there exists an integer $k \geqq 1$ such that

$$
a_{k}-\epsilon\left(a_{k}\right)<\delta_{j+1}<\delta_{j-1}<a_{k}+\epsilon\left(a_{k}\right) .
$$

Now we define distinct positive numbers $\left\{\delta_{i j}\right\}_{i, j=1}^{\infty}$ as follows. Fix $j \geqq 1$. Define $\delta_{1 j}=\delta_{j}$. For $i>1$ choose the $\delta_{i j}$ 's to be distinct numbers strictly between $\delta_{j}$ and $\delta_{j+1}$.

Now define

$$
\begin{aligned}
& E_{i 1}=\left\{x \in X_{n}: \rho(x, A) \geqq \delta_{i 1}\right\}, \quad(i \geqq 1) ; \\
& E_{i j}=\left\{x \in X_{n}: \delta_{i j} \leqq \rho(x, A) \leqq \delta_{i, j-1}\right\}, \quad(i \geqq 1, j>1) ; \\
& C_{i j}=C_{i} \cap E_{i j}, \quad C_{i j}^{\prime}=C_{i}^{\prime} \cap E_{i j}, \quad(i, j \geqq 1) .
\end{aligned}
$$

3.5. Assertion 2. There exists a $\tau>0$ such that $\rho\left(C_{i j}, C_{i j}^{\prime}\right) \geqq \tau$ for $i, j \geqq 1$.

Proof. Define $\tau=\min \left\{\gamma, \epsilon \delta_{2}^{2} / 4 N\right\}$.

Case 1. $j=1$. Suppose there exists an integer $i \geqq 1$ such that 
$\rho\left(C_{i 1}, C_{i 1}^{\prime}\right)<\tau$. Let $x \in C_{i 1}$ and $y \in C_{i 1}^{\prime}$ be such that $\rho(x, y)<\tau$. Note that $\rho(x, A)>\delta_{2}$ and $\rho(y, A)>\delta_{2}$, since $\{x, y\} \subset E_{i 1}$. Hence

$$
\begin{aligned}
\sigma^{N}(x, y) & \leqq \rho(x, y)+\sum_{i=1}^{N} \frac{\left|\rho\left(x, A_{i}\right)-\rho\left(y, A_{i}\right)\right|}{\rho\left(x, A_{i}\right) \cdot \rho\left(y, A_{i}\right)} \\
& \leqq \rho(x, y)+\sum_{i=1}^{N} \frac{\rho(x, y)}{\rho\left(x, A_{i}\right) \cdot \rho\left(y, A_{i}\right)} \\
& <\tau+N \tau / \delta_{2}^{2} \\
& <\epsilon / 4+\epsilon / 4=\epsilon / 2,
\end{aligned}
$$

a contradiction to (1).

Case 2. $j>1$. Fix $i \geqq 1$ and $j>1$. Now by the definition of $E_{i j}$ and by (2),

$$
\begin{aligned}
E_{i j} & \subset\left\{x \in X_{n}: \delta_{j+1} \leqq \rho(x, A) \leqq \delta_{j-1}\right\} \\
& \subset S(\epsilon(a))
\end{aligned}
$$

for some $a$ such that $0<a \leqq \delta / 4$. Therefore by the definitions of $C_{i j}$ and $C_{i j}^{\prime}$ and Assertion $1, \rho\left(C_{i j}, C_{i j}^{\prime}\right) \geqq \gamma \geqq \tau$.

3.6. Lemma [7]. Let $X$ be a topological space, let $C$ and $C^{\prime}$ be disjoint closed sets in $X$, and let $\left\{D_{j}\right\}_{j=0}^{\infty}$ be an open cover of $X$ such that $D_{0}=\varnothing$ and $\bar{D}_{j} \subset D_{j+1}$ for all $j \geqq 1$. Suppose there exist closed sets $\left\{B_{j}\right\}_{j=1}^{\infty}$ in $X$ such that $B_{j} \subset \bar{D}_{j}-D_{j-1}$ for $j \geqq 1$ and $B_{j}$ separates $C \cap\left(\bar{D}_{j}-D_{j-1}\right)$ and $C^{\prime} \cap\left(\bar{D}_{j}-D_{j-1}\right)$ in $\bar{D}_{j}-D_{j-1}$ for $j \geqq 1$. Then there exists a closed set $B$ in $X$ such that $B$ separates $C$ and $C^{\prime}$ in $X$ and $B \subset \cup_{j=1}^{\infty}\left(B_{j} \cup\left(\bar{D}_{j}-D_{j}\right)\right)$.

3.7. Conclusion of the proof of the theorem. By Assertion 2 and the equality $d_{5}\left(X_{n}, \rho\right)=[n / 2]$, there exist closed sets $\left\{B_{i j}^{\prime}\right\}_{i, j=1}^{\infty}$ in $X_{n}$ such that $B_{i j}^{\prime}$ separates $C_{i j}$ and $C_{i j}^{\prime}$ in $X_{n}$ for $i, j \geqq 1$ and order $\left\{B_{i j}^{\prime}\right\}_{i, j=1}^{\infty} \leqq[n / 2]$. For $i \geqq 1$ define $D_{i 0}=\varnothing$. For $i, j \geqq 1$ define $D_{i j}$ $=\left\{x \in X_{n}: \rho(x, A)>\delta_{i j}\right\}$ and $B_{i j}=B_{i j}^{\prime} \cap\left(\bar{D}_{i j}-D_{i, j-1}\right)$, where for every $i$ and $j$ the closure of $D_{i j}$ is taken with respect to $X_{n}$. Then clearly $B_{i j}$ separates $C_{i}$ and $C_{i}^{\prime}$ in $\bar{D}_{i j}-D_{i, j-1}$ for $i, j \geqq 1$ and

$$
\operatorname{order}\left\{B_{i j}\right\}_{i, j=1}^{\infty} \leqq[n / 2] \text {. }
$$

Now fix $i \geqq 1$. Clearly $X_{n}, C_{i}, C_{i}^{\prime},\left\{D_{i j}\right\}_{j=0}^{\infty}$, and $\left\{B_{i j}\right\}_{j=1}^{\infty}$ satisfy the conditions of the lemma. Therefore there exists a closed set $B_{i}$ in $X_{n}$ such that $B_{i}$ separates $C_{i}$ and $C_{i}^{\prime}$ in $X_{n}$ and

$$
B_{i} \subset \bigcup_{j=1}^{\infty}\left(B_{i j} \cup\left(\bar{D}_{i j}-D_{i j}\right)\right)
$$


But for $j \geqq 1$,

$$
\bar{D}_{i j}-D_{i j} \subset\left\{x \in X_{n}: \rho(x, A)=\delta_{i j}\right\} .
$$

Hence

$$
B_{i} \subset \bigcup_{j=1}^{\infty}\left(B_{i j} \cup\left\{x \in X_{n}: \rho(x, A)=\delta_{i j}\right\}\right) .
$$

Therefore, by (3) and the fact that the $\delta_{i j}$ 's are distinct for $i, j \geqq 1$, we have that order $\left\{B_{i}\right\}_{i=1}^{\infty} \leqq[n / 2]+1$, and the proof is complete.

\section{REFERENCES}

1. E. E. Grace (editor), Topology conference, Arizona State University, Tempe, Ariz., 1968. MR 38 \#5152.

2. John G. Hocking and Gail S. Young, Topology, Addison-Wesley, Reading, Mass., 1961. MR 23 \#A2857.

3. Richard E. Hodel, Note on metric-dependent dimension functions, Fund. Math. 61 (1967), 83-89. MR 36 \#3326.

4. M. Katětov, On the relation between metric and topological dimensions, Czechoslovak Math. J. 8 (83) (1958), 163-166. (Russian) MR 21 \#3830.

5. K. Nagami and J. H. Roberts, Metric-dependent dimension functions, Proc. Amer. Math. Soc. 16 (1965), 601-604. MR 33 \#3264.

6. - A study of metric-dependent dimension functions, Trans. Amer. Math. Soc. 129 (1967), 414-435. MR 35 \#6131.

7. J. H. Roberts, Dimension function $d_{2}$ and covering dimension, Duke Math. J. (to appear).

8. K. Sitnikov, Example of a two-dimensional set in three-dimensional Euclidean space allowing arbitrarily small deformations into a one-dimensional polyhedron and a certain new characteristic of the dimension of sets in Euclidean spaces, Dokl. Akad. Nauk SSSR 88 (1953), 21-24. (Russian) MR 14, 894.

DUKE UNIVERSITY 